\title{
Invasive papillary carcinoma of the breast: a rare case report
}

\author{
Pratibha Issar ${ }^{*} \mathbb{C}$, M. Ravindranath and Manish Dewangan
}

\begin{abstract}
Background: Invasive Papillary Carcinomas (IPC) are rare and account for approximately $0.5 \%$ of all invasive breast carcinomas. Most of them are seen in post-menopausal women and have a good prognosis. These tumors lack the myoepithelial cell layer (MCL) within the papillae or at the periphery of the tumor with areas showing stromal invasion or invasion into lymphovascular spaces. Immunohistochemistry (IHC) for myoepithelial cells and basement membrane is essential for the diagnosis of invasive cancer.

Case presentation: We present a rare case of IPC in a 74-year-old woman who presented with complaints of gradually increasing painless retroaerolar mass in the left breast of two months duration. The mass was irregular, having an oblong as well an adjacent high density mass lesion on mammography. Ultrasound (US), and Magnetic Resonance Imaging (MRI) helped in the diagnosis of the possibility of a malignant breast lesion. Left-sided modified radical mastectomy was performed and the specimen was histopathologically diagnosed as Invasive Papillary carcinoma. Immunohistochemistry confirmed the diagnosis.
\end{abstract}

Conclusions: Invasive Papillary Carcinomas of the breast are rare cancers in post-menopausal women. We have highlighted the role of Mammography, US, and MRI in early diagnosis so that timely management is possible.

Keywords: Invasive papillary carcinoma, Ultrasound, Magnetic resonance imaging, Immunohistochemistry

\section{Background}

Invasive Papillary Carcinoma is a rare malignant tumor of the breast having a much better survival rate as compared to other carcinomas. Bloody nipple discharge is a relatively common presenting sign, occurring in $22-34 \%$ of cases or may present as a palpable mass. Though the frequency of axillary node metastasis is low, treatment often involves mastectomy and axillary node dissection. Histologically lesions are classified as papillary carcinomas having a frond-forming growth pattern supported by a fibrovascular stalk. The absence of the myoepithelial layer differentiates carcinomas from benign papillary lesions $[1,2]$. We report this case because of its rarity and typical imaging finding leading to the diagnosis of IPC.

${ }^{*}$ Correspondence: pratibhaissar@gmail.com

J.L.N Hospital and Research Centre, Bhilai, Chhattisgarh 490009, India

\section{Case presentation}

A 74-year-old postmenopausal woman presented with complaints of gradually increasing painless retroaerolar mass in the left breast, upper outer quadrant of two months duration. She did not have any history of nipple discharge or a family history of breast cancer.

On examination, an oblong mass was palpable in the retroaerolar region at 1 O'clock position and it was extending towards the periphery. No nipple retraction or discharge was observed. Examination of the bilateral axillae revealed no palpable lymph nodes.

A diagnostic mammogram was performed on Alpha -RT (GE) showed heterogeneous dense breast with an irregular, high-density mass having oblong component noted in retroaerolar region in the center-left breast involving anterior and middle depth with indistinct margin. An irregular hyperdense mass mass with indistinct margins was also seen in the upper outer quadrant adjacent to the above-mentioned oblong structures 


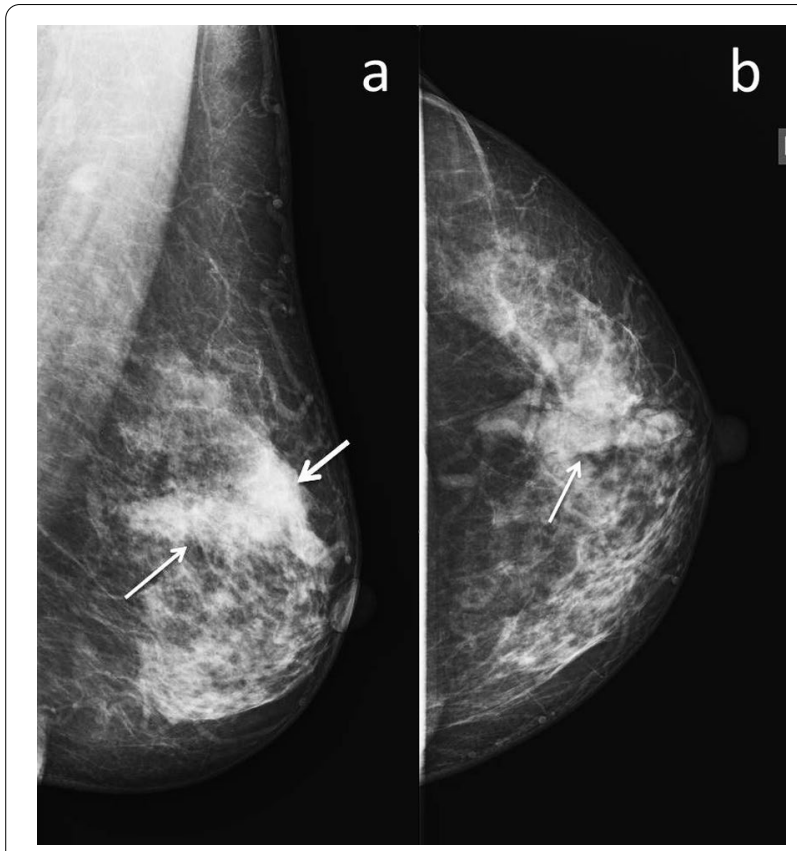

Fig. 1 Mediolateral oblique (a) and Cranio caudal (b) mammography of the left breast showing high-density mass having an oblong (thin arrow) as well as an irregular mass component (thick arrow) in retroaerolar as well as an upper outer quadrant, involving anterior and middle depth
(Fig. 1a, b). No intra-mass calcification, skin thickening, or nipple retraction were seen. Nonspecific normal size lymph nodes were seen in the axilla. BIRADS 4 category was assigned to the left breast mass on mammography. Breast ultrasound (US) was performed on Samsung RS $80 \mathrm{~A}$, using a linear probe of $4-18 \mathrm{MHz}$ with an elastography facility. The US revealed a solitary dilated duct $(1.5 \mathrm{~cm})$ for a length of $6.6 \mathrm{~cm}$, seen at 1 O'clock of the retroaerolar region. The lumen of the duct was filled with a hypoechoic mass showing increased vascularity (Fig. 2a). There was a corresponding loss of linear echogenicity of the ductal wall. An irregular $(2.4 \times 2.1 \mathrm{~cm})$ heterogeneous, hypoechoic solid mass with micro lobulated margin and posterior acoustic enhancement was seen at 12 O'clock position adjacent to the dilated duct and was communicating with the duct (Fig. 2b). The mass was showing increased vascularity with an E-Strain of 3.17. Bilateral benign axillary lymph nodes were noticed. Right breast parenchyma was normal. The breast US was graded as BIRADS $4 \mathrm{C}$.

Plain and contrast Magnetic Resonance Imaging (MRI) of the breast was performed, for further management as well as to see the extent of the mass. MRI of the breast was done on a 1.5Tesla MR system (GE Signa Excite) using a dedicated 8 channel breast coil. On MRI examination bilateral breast tissue was heterogeneous fibro glandular in nature with minimal background parenchymal

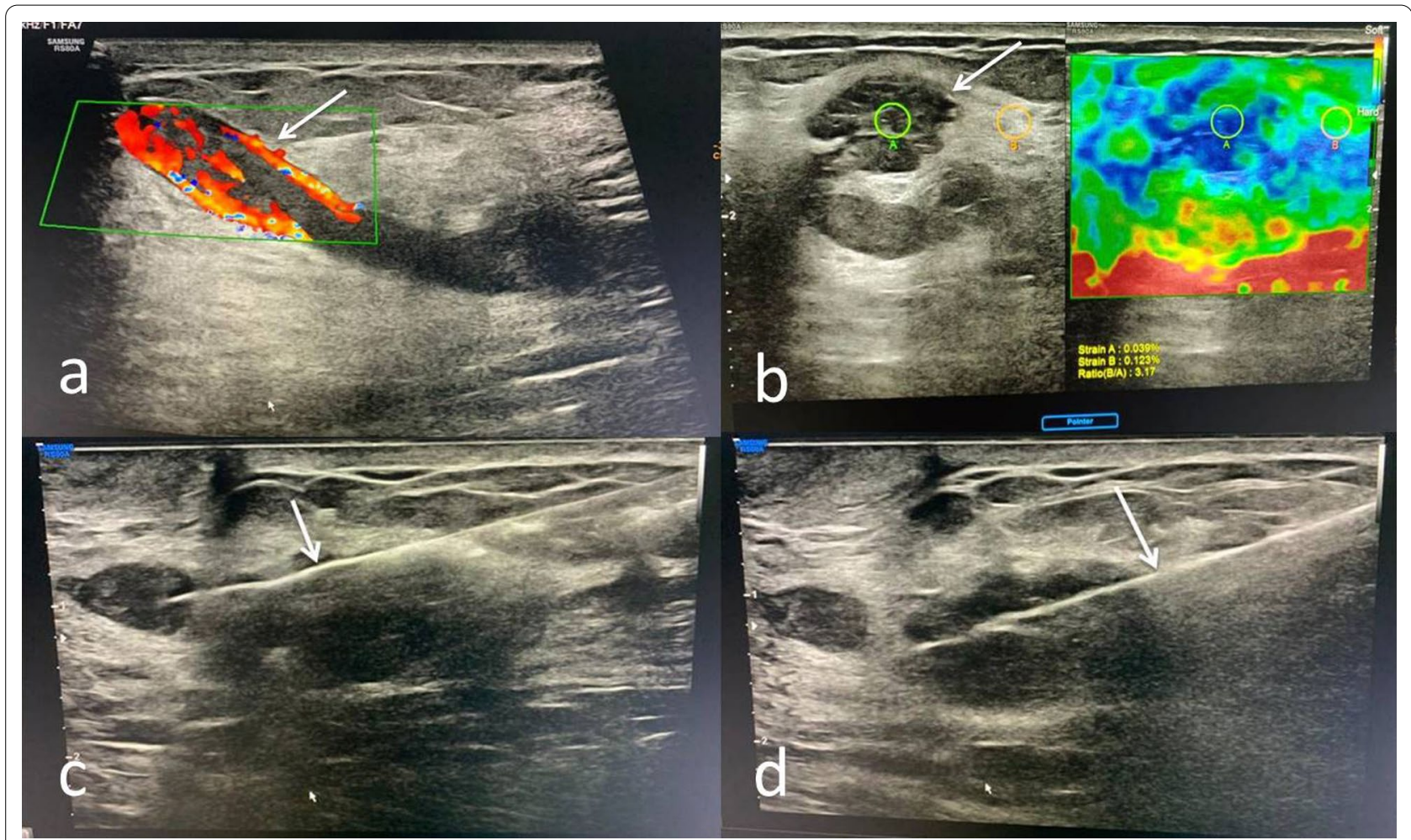

Fig. 2 Ultrasonography demonstrates dilated duct filled with a solid mass (arrow) showing increased vascularity (a) adjacent heterogenous hypoechoic mass with E -Strain of 3.17 (b), biopsy needle (arrow) seen within ductal (c), and associated mass (d) 


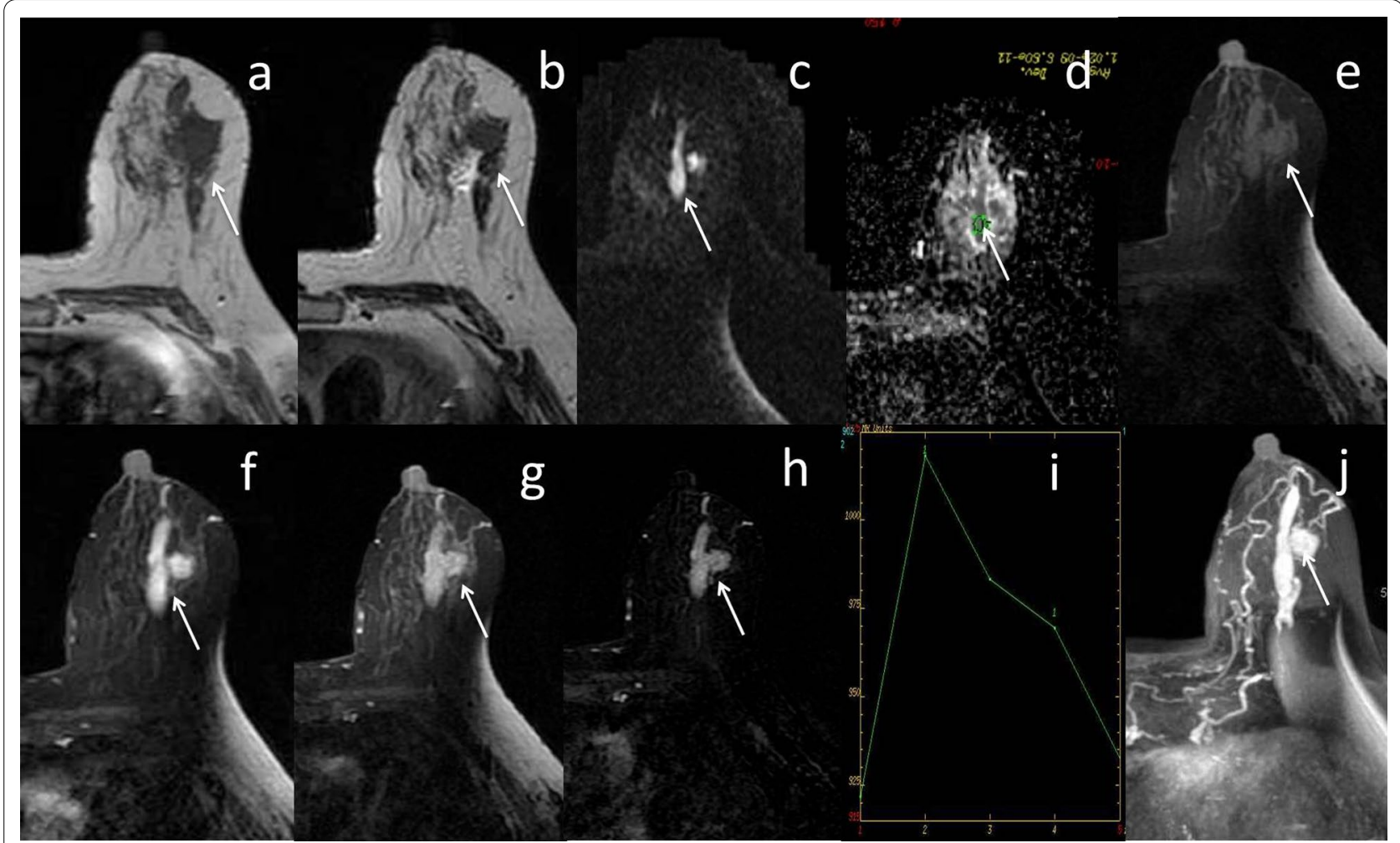

Fig. 3 The mass (arrow) appears hypointense on T1 (a), hypointense with perilesional edema on T2 weighted images (b), showing diffusion restriction (c) with hypointensity (arrow) on ADC map (d), pre-contrast (e), early (f), and delayed ( $\mathbf{g}$ ) post-contrast images along with subtraction images (h) showing early contrast uptake and delayed washout. Type 3 kinetic curve seen (i) along with the MIP image (j) showing a mass with increased vascularity

enhancement. An irregular T1, T2 hypointense mass having an oblong component measuring $6.8 \times 1.5 \mathrm{~cm}$ seen at 12 to 1 O'clock position extending $1.8 \mathrm{~cm}$ away from nipple to middle depth posteriorly (Fig. 3a, b). The oblong mass was showing diffusion restriction with an ADC value of $1.10^{\mathrm{e}-09}$. An irregular shape $(2.4 \times 2.2 \mathrm{~cm})$, irregular margin T1, T2 hypointense mass was noticed connected with the mid-lateral aspect of above mention oblong mass. This mass was also showing diffusion restriction with an ADC value $1.22^{\mathrm{e}-09}$ (Fig. 3c, d). Few surrounding T2 and T2 FAT SAT hyperintensities were seen suggestive of edema. Both, the oblong ductal component as well as the connected solid mass were showing heterogenous early contrast enhancement with washout in delayed phases and showing type III kinetic curve (Fig. 3e-j). These findings point towards a BIRADS 5 lesion in the left breast. So based on Mammography, Ultrasound, and MRI findings a diagnosis of malignant breast mass was made. Consequently, a tru-cut biopsy of the ductal, as well as adjacent mass, performed under ultrasound guidance (Fig. 2c, d). Histopathological examination revealed multiple linear cores displaying tumor cells infiltrating the fibro collagenous tissue arranged in a predominantly papillary pattern, nests, and tubules. Individual cells are round to oval with hyperchromatic nuclei, inconspicuous nucleoli, and moderate cytoplasm. On IHC tumor cells were positive for CK7, GATA 3, and negative for CK 20, CEA, P63, Calponin, S-100 P. Hormonal receptor studies showed ER strong positive (95\%) PR- moderate positive, Her-2/Neu negative, and a Ki -67 score of $10 \%$. All the above features were suggestive of Invasive Papillary Carcinoma. The patient underwent a modified radical mastectomy. IPC was confirmed on the surgical specimen (Fig. 4).

\section{Discussion}

Papillary Breast Carcinomas are rare, seen predominantly in postmenopausal women between the sixth and eighth decade, and have a good prognosis. Clinically they can be asymptomatic or present with bloody nipple discharge,or an abnormal palpable mass. Mammography can show a round, oval or irregular high-density mass, sometimes associated with satellite nodules, and margins are circumscribed but may be obscured or indistinct. Accompanying microcalcification or a dilated ductal pattern may be present [2]. 


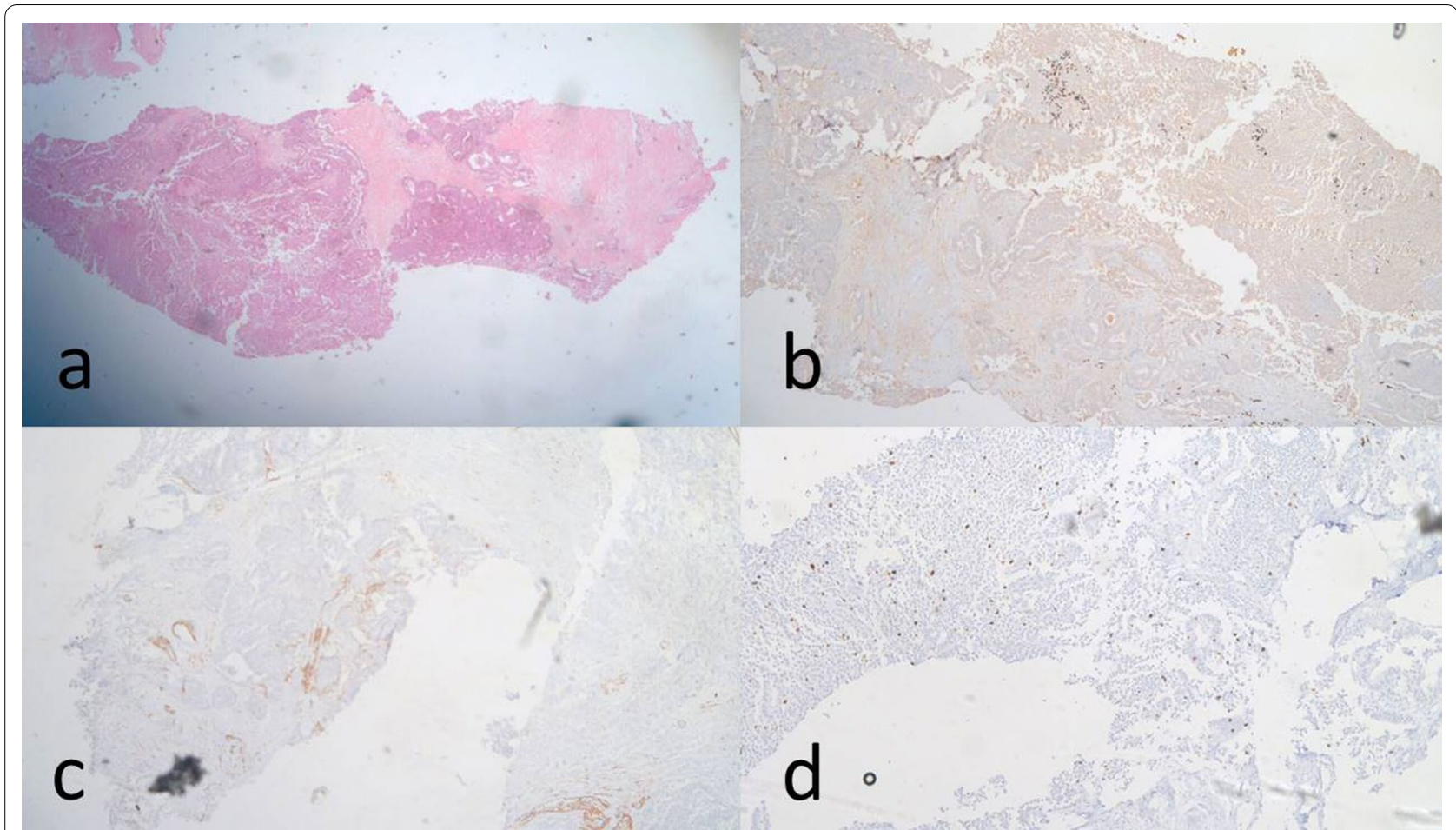

Fig. 4 The tumor cells showing papillary pattern (a). Immunohistochemistry showing tumor cells negative for P63 (b), calponin (c), with a Ki67 score of $10 \%($ d)

Ultrasonic evaluation of Invasive Papillary Carcinoma reveals a hypoechoic solid mass commonly accompanied with posterior acoustic enhancement. Complex cystic and solid masses with increased vascularity in the solid area can be seen. There can be associated ductal dilation with intra ductal solid mass [3].

Limited experience is available for Magnetic Resonance Imaging of IPC. On MRI, papillary carcinomas may appear as irregular enhancing nodules or enhancing complex cysts. Morphological features, as well as kinetic curves of IPC, are variable on MRI. MRI helps in the preoperative mapping of multiple papillary lesions, thereby facilitating optimal surgical planning [4].

Fine needle aspiration cytology [FNAC] reveals atypical cells in the smear. Sonography-guided vacuum-assisted core biopsy is a much better option than aspiration cytology. The gun biopsy mainly hits the solid center of the tumor and the invasive component can only be recognized at the periphery of the tumor, so excisional biopsy of papillary lesions is an effective approach to demonstrate invasion [5]. Malignant papillary neoplasm of the breast includes several microscopically distinct lesions such as DCIS (Ductal Carcinoma in Situ) arising in intra ductal papilloma, papillary DCIS, encapsulated papillary carcinoma, solid papillary carcinoma, and invasive papillary carcinoma. All malignant papillary lesions of the breast lack an intact myoepithelial cell layer (MCL) within the papillae or at the periphery of the tumor [6].

IHC is very useful in the assessment of myoepithelial cells and basement membrane for the diagnosis of invasive cancers. The Myoepithelial cells are absent in invasive cancers. There are many known myoepithelial markers such as S-100, Calponin, CD 1O, smooth muscle myosin heavy chain, alpha-smooth muscle actin, P63, and high molecular weight Cytokeratin with different sensitivities and specificities. Of these P63 and smooth muscle, myosin is more specific. A special myoepithelial marker P63 stains the cell muscles only [2]. Genetic Features may help in distinguishing papillary carcinoma from benign lesions. Loss of heterozygosity (LOH) of $16 \mathrm{q} 23$ is specific to the malignant lesion. Alternation in chromosomes 3,7,17 and $\mathrm{X}$ using multicolor fluorescence in situ hybrididization was noticed in DNA-aneuploid carcinoma. So DNA - ploidy in association with changes in specific loci helps in the diagnosis of papillary carcinoma [1]

The overall prognosis of Invasive Papillary Carcinoma is better than other breast malignancies such as infiltrating duct carcinoma. Treatment options are wide local excision, with or without adjuvant radiotherapy or chemotherapy. Tamoxifen is an important drug as this cancer 
seems to be almost certainly hormonal positive and HER -2 negative $[7,8]$.

According to Hesham and Almohamady, malignancy is more common if an Intraductal mass fills the duct and extends outside the duct with a distance from the nipple more than $1.5 \mathrm{~cm}$. In our case also the mass was filling the duct as well as extending outside the duct and was $1.8 \mathrm{~cm}$ away from the nipple thus favoring the malignant nature of the mass [9]. Ultrasound and MRI can help in making a diagnosis of Invasive Papillary Carcinoma of the breast.

\section{Conclusions}

We would like to emphasize on the rare presentation of this Invasive Papillary Carcinoma as an Intraductal mass filling the dilated duct along with extension outside the duct as a solid mass lesion. Invasive Papillary Carcinoma carries an excellent prognosis, but diagnosis remains challenging, and IHC for myoepithelial cell markers helps in its proper management.

\section{Abbreviations}

IPC: Invasive papillary carcinoma; US: Ultrasound; MRI: Magnetic resonance imaging; IHC: Immunohistochemistry; MCL: Myoepithelial cell layer; FNAC: Fine needle aspiration cytology.

\section{Acknowledgements}

We would like to thank Mr. Arnesh Kumar Issar for his help with the Manuscript preparation, review and submission.

\section{Authors' contributions}

Pl: Conception, design of work, acquisition, analysis \& interpretation of data. RM: Analysis (Pathology \& Immunohistology) \& manuscript revision. MD: Design of work \& insights into treatment procedure (performing surgeon). All authors have read and approved the manuscript.

\section{Funding}

Not applicable.

\section{Availability of data and materials}

Yes, the datasets used and or analyzed during the current study are available from the corresponding author on reasonable request.

\section{Declarations}

Ethics approval and consent to participate

Not applicable, as no personal details of the participants are presented.

\section{Consent for publication}

Written informed consent to publish this information was obtained from the study participant.

\section{Competing interests}

The authors declare that they have no competing interests.

Received: 11 May 2021 Accepted: 23 September 2021

Published online: 08 October 2021

References

1. Pal SK, Lau SK, Kruper L et al (2010) Papillary carcinoma of the breast: an overview. Breast Cancer Res Treat 122(3):637-645

2. Vani D, Geetanjali S, Punja GM, Bharathi M (2015) A case of invasive papillary breast carcinoma: Fierce Façade with favorable prognosis. J Can Res Ther 11:1029

3. Brown A, Gupta P, Singh S et al (2016) Invasive solid papillary carcinoma breast. A rare case report. Int J Adv Res 4(12):1301-1305

4. Zhang L, Zhuang L, Chang S et al (2017) A pilot evaluation of magnetic resonance imaging characteristic seen with solid Papillary carcinoma of the breast in 4 patients. BMC Cancer 17:525

5. Ingle SB, Murdeshwar HG, Siddique S (2016) Papillary carcinoma of the breast: mini review. World J Clin Cases 4(1):20-24

6. Nunez DL, Gonzalaz FC, Monica C et al (2020) Papillary lesion of the breast: a review. Breast Cancer Manag Sept 9(4):52

7. Salemis NS, Mourtzoukou D (2021) Encapsulated papillary carcinoma of the breast. Breast 27:280-283

8. Bhosale SJ, Kshirsager AV, Sulhyan SR et al (2010) Invasive papillary breast carcinoma. Case Rep Oncol 3:410-415

9. Sheikh HE, Almetaher HA, Hamdy S et al (2015) Intraductal breast masses: sonographic and mammographic predictors of malignancy. Egypt J Radiol Nuclear Med 46:1271-1278

\section{Publisher's Note}

Springer Nature remains neutral with regard to jurisdictional claims in published maps and institutional affiliations.

\section{Submit your manuscript to a SpringerOpen ${ }^{\odot}$ journal and benefit from:}

- Convenient online submission

$\checkmark$ Rigorous peer review

- Open access: articles freely available online

- High visibility within the field

- Retaining the copyright to your article

Submit your next manuscript at springeropen.com 